\title{
Fidelity and Quantum phase transition for the Heisenberg chain with the next-nearest-neighbor interaction
}

\author{
Shu Chen, ${ }^{1}$ Li Wang, ${ }^{1}$ Shi-Jian $\mathrm{Gu},{ }^{2}$ and Yupeng Wang ${ }^{1}$ \\ ${ }^{1}$ Beijing National Laboratory for Condensed Matter Physics, \\ Institute of Physics, Chinese Academy of Sciences, Beijing 100080, China \\ ${ }^{2}$ Department of Physics and Institute of Theoretical Physics, \\ The Chinese University of Hong Kong, Hong Kong, China
}

(Dated: October 22, 2018)

\begin{abstract}
In this paper, we investigate the fidelity for the Heisenberg chain with the next-nearest-neighbor interaction (or the $J_{1}-J_{2}$ model) and analyze its connections with quantum phase transition. We compute the fidelity between the ground states and find that the phase transition point of the $J_{1}-J_{2}$ model can not be well characterized by the ground state fidelity for finite-size systems. Instead, we introduce and calculate the fidelity between the first excited states. Our results show that the quantum transition can be well characterized by the fidelity of the first excited state even for a small-size system.
\end{abstract}

PACS numbers: 64.60.-i, 03.67.-a, 05.70.Fh, 75.10.-b

Quantum phase transitions (QPTs) driven by purely quantum fluctuations have been extensively studied in the recent years [1]. One of the research focuses in the cross field of quantum many-body theory and quantuminformation theory is the application of quantum entanglement to the analysis of QPTs [2, 3]. The intriguing issue of the role of quantum entanglement in characterizing QPTs has been investigated for different many-body systems [2, 3, 4, 5, 6, 7]. More recently, the ground state fidelity or the overlap between two ground states corresponding to two slightly different values of the external parameters is proposed to characterize QPTs [8, [9]. Within examples of the Dicke and XY models, it has been shown that the ground state fidelity shows a dramatic drop in the vicinity of the QPT point of the system. Similar to the quantum entanglement, the notation of fidelity is also borrowed from the field of quantum information science. Being a pure geometrical quantity, an obvious advantage of the fidelity is that it can be a promising candidate to characterize the QPT because no a priori knowledge of the order parameter and the symmetry of the system is needed [9]. By using the fidelity as a measure, Buonsante et al. can determine the quantum phase transition point of the Bose-Hubbard model which is hard to be characterized by the quantum entanglement [10].

Despite the success of the ground state fidelity 8, 9, 10, 11, 12, 13] as a measure of QPTs in several concrete examples, it is still not clear whether the effectiveness of the ground state fidelity in the study of QPT is general for most of the many-body systems [12]. One of the obstacles lies in the difficulty in the calculation of ground state fidelity because it is generally very hard to analytically obtain the ground state wavefunction of a manybody system except a few examples. An even more basic question is whether the ground state fidelity is a modelindependent indicator for QPTs which exhibits qualitatively different behaviors at and off the transition point?

In this paper, we will show that the ground state fi- delity is not always a good characterization of the regions of criticality that define QPTs for a one-dimensional Heisenberg system with next-nearest-neighbor coupling. Instead, we find that the overlap of the first excited state or the fidelity of the first excited state shows a dramatic drop in the vicinity of the QPT point of the system and can be used to characterize the QPT. We note that our conclusions are based on the finite size of the chain considered (up to 24 sites) which may not exclude that the ground state fidelity for very large systems could in principle be a characterization of quantum phase transition just like in the case of XY model [9]. However, in the current computation sources it is not practical to compute a non-integrable spin system to a very large size as in the case of the exactly solvable XY model.

The Hamiltonian of a one-dimensional Heisenberg chain with the next-nearest-neighbor coupling reads

$$
H(\lambda)=\sum_{j=1}^{L}\left(\hat{s}_{j} \hat{s}_{j+1}+\lambda \hat{s}_{j} \hat{s}_{j+2}\right),
$$

where $\hat{s}_{j}$ denotes the spin- $1 / 2$ operator at the $j$ th site, $L$ denotes the total number of sites, and the periodic boundary conditions $\hat{s}_{1}=\hat{s}_{L+1}$ are assumed. The only effective parameter $\lambda$ refers to the ratio between the next-nearest-neighbor (NNN) coupling and the nearestneighbor (NN) coupling. This model is invariant under a global $\mathrm{SU}(2)$ rotation, which implies total spin conservation. For a general $\lambda$, the model is not analytically solvable. When $\lambda=0$, the model is exactly solvable by Bethe-ansatz method [14, 15]. When $\lambda=1 / 2$, the model reduces to the Majumda-Ghosh model whose ground state is a uniformly weighted superposition of the two nearest-neighbor valence bond states [16].

The ground-state properties of the model (1) has been widely studied by analytical method, such as bosonization and effective field theory [17, 18], and numerical method, such as exact diagonalization [19, 20] and density matrix renormalization group [21, 22, 23]. The quan- 


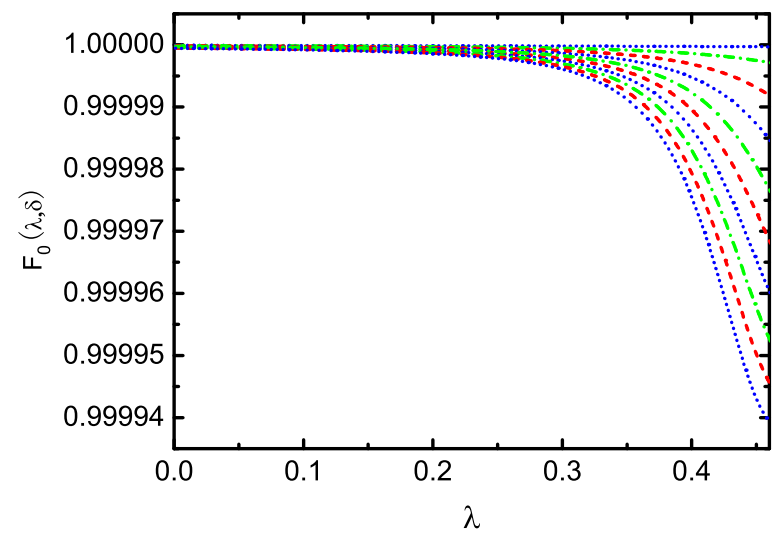

FIG. 1: (Color online). Ground state fidelity $F_{0}(\lambda, \delta)$ as a function of $\lambda$. The lines from top to bottom correspond to Heisenberg chains with sizes $L=$ $6,8,10,12,14,16,18,20,22,24$, respectively.

tum phase transition driven by the frustration (the competition between the NN and NNN interaction) is well understood for general $\lambda$. Frustration due to $\lambda$ is irrelevant when $\lambda<\lambda_{c}$, and the system renormalizes to the Heisenberg fixed point, whose ground state is described as a spin fluid or Luttinger liquid with massless spinon excitations. As $\lambda>\lambda_{c}$, the frustration term is relevant and the ground state flows to the dimerized phase with a spin gap open. The transition from spin fluid to dimerized phase is known to be of BeresinskiiKosterlitz-Thouless (BKT) type [17, 18, 24, 25]. It has been difficult to determine the BKT point numerically due to the problem of logarithmic correction [26]. The critical value of $\lambda_{c}=0.2411 \pm 0.0001$ has been accurately determined by numerical methods and conformal field theory method [27, 28]. The entanglement for the model (11) has been studied in Ref. [5] where the ground concurrences between the nearest-neighbors and the nextnearest-neighbors are calculated as functions of $\lambda$. No singularities of the concurrences around $\lambda_{c}$ are found for the system with different sizes, which implies that the concurrences may be not an effective characterization of the QPT. Very recently, Chhajlany et. al. found that there is a deviation from the scaling behavior of the entanglement entropy characterizing the unfrustrated Heisenberg chain when $\lambda=J_{2} / J_{1}>0.25$ and thus concluded that this feature can be used as an indicator of the dimer phase transition [7].

In the present work, we will study the features of the fidelity for the model (1) and focus on the regime of $0<$ $\lambda<0.5$ in which the BKT-type quantum phase transition happens. Following Ref. [9], the ground state fidelity is defined as the overlap between $\left|\Psi_{0}(\lambda)\right\rangle$ and $\left|\Psi_{0}(\lambda+\delta)\right\rangle$,

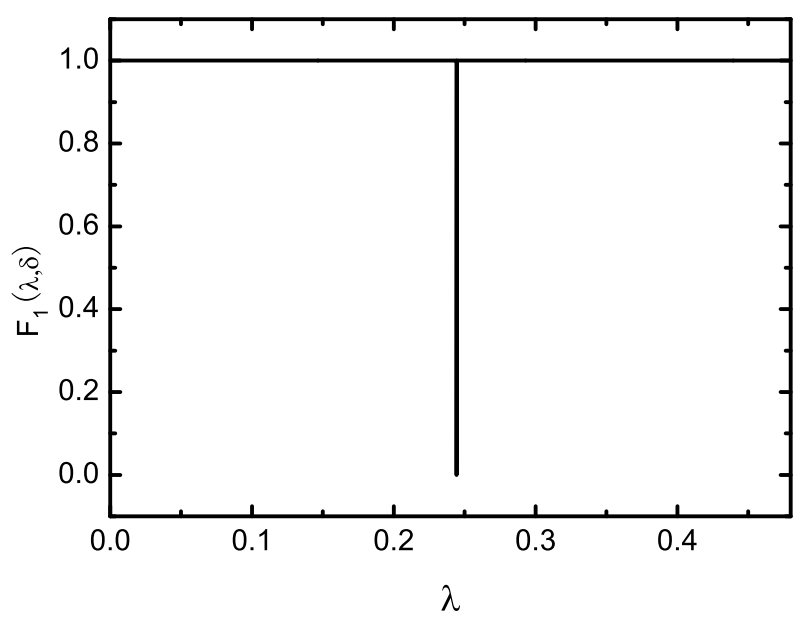

FIG. 2: The fidelity of the first excited state $F_{1}(\lambda, \delta)$ as a function of $\lambda$ for a Heisenberg chain composed of 10 spin sites.

i.e.

$$
F_{0}(\lambda, \delta)=\left|\left\langle\Psi_{0}(\lambda) \mid \Psi_{0}(\lambda+\delta)\right\rangle\right|,
$$

where $\Psi_{0}(\lambda)$ is the ground state wavefunction of Hamiltonian (11) corresponding to the parameter $\lambda$ and $\delta$ is a small quantity. In general, one can numerically solve the eigenvalue problem of the Hamiltonian and obtain the eigenfunctions by using the exact diagonalization method for a finite-size system.

We calculate the ground state fidelity of the Heisenberg chain given by Eq. (1) for different sizes. In Fig. 1. we plot the ground state fidelity as a function of $\lambda$ with $\delta=1.7 \times 10^{-3}$ for the frustrated Heisenberg chain with sizes of $L=6,8,10,12,14,16,18,20,22,24$. We observe that the ground state fidelity is almost a constant and equal to unity for a wide range of the parameter $0<\lambda<0.5$. According to [9], one expect a sharp drop of the ground state fidelity to characterize the critical point of the QPT. However, for the present model, no a sharp drop in the ground state fidelity is detected in the regime under investigation for the systems with size up to 24 sites. Also, we don't find any peaks in the derivatives of the ground state fidelity, which we do not show here. We note that no exact analytical results are available for the present $J_{1}-J_{2}$ model except the special case of $J_{2}=0$ and $J_{2} / J_{1}=0.5$. Therefore, we have to calculate the ground state wavefunctions as well as the ground state fidelity by using the numerical exact diagonalization method which however limits the size of our investigated system. Nevertheless, our results imply that critical points of the quantum phase transitions can not be well characterized by the ground state fidelity for a finite size system. 


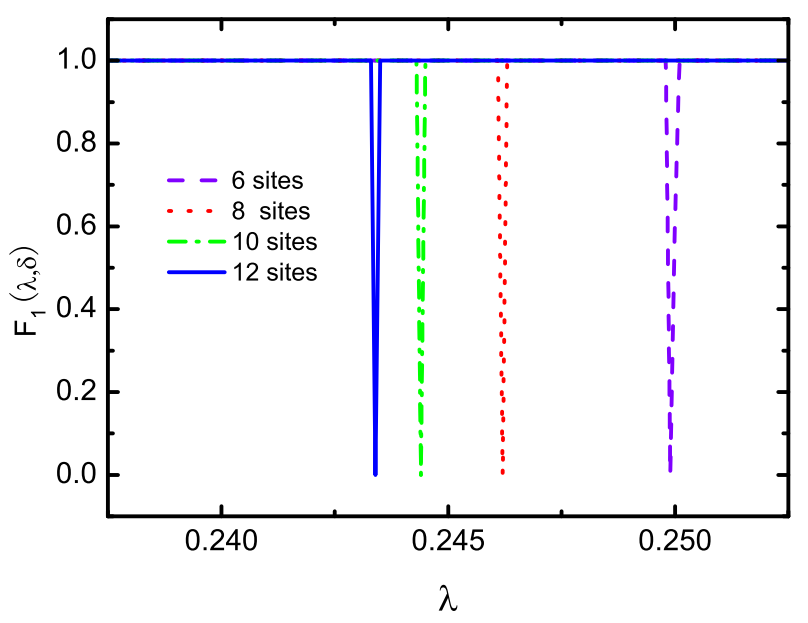

FIG. 3: (Color online). The fidelity of the first excited state $F_{1}(\lambda, \delta)$ as a function of $\lambda$. Different colors correspond to Heisenberg chains composed of different numbers of spin sites.

We recall that, in the scheme of field theory method, the phase transition point for the model (11) is determined by the opening of the elementary excitation gap [17], which implies that the excited states play an important role in determining the phase diagram of the system. Therefore, it is instructive to investigate the fidelity of the first excited state of the Heisenberg chain (11). Similarly, the fidelity of the first excited state of the system is defined as the overlap of the first excited states with parameter $\lambda$ and $\lambda+\delta$,

$$
F_{1}(\lambda, \delta)=\left|\left\langle\Psi_{1}(\lambda) \mid \Psi_{1}(\lambda+\delta)\right\rangle\right|
$$

where $\Psi_{1}(\lambda)$ represents the first excited state of the system. We first calculate the first excited state fidelity of a Heisenberg chain with $L=10$ as shown in Fig. 2, Obviously, there is a sudden drop in the first excited state fidelity at the point a little smaller than $\lambda=0.25$. This lights our hope and convinces us that the first excited state fidelity may be a good candidate to characterize the critical point between the spin fluid phase and the dimerized phase. From this point of view, we continue to calculate the first excited state fidelity of the $J_{1}-J_{2}$ model for cases: $L=6,8,12$. Fig. 3 shows the behavior of the first excited state fidelity $F_{1}(\lambda, \delta)$ with $\delta=1.7 \times 10^{-4}$ as a function of $\lambda$ for the systems with different sizes. The extrema of the first excited state fidelity feature a scaling behavior. The size dependence of the critical point $\lambda_{c}$ versus $1 / L^{2}$ is shown in Fig. 4. The four dots correspond to the four cases: $L=12,10,8,6$. We make a polynomial fit to the four dots. And we find out that when it comes to the case: $L \rightarrow \infty$, the critical point is $\lambda_{c}=0.24107$. This consists with the value $\lambda_{c}=0.2411 \pm 0.0001$ given by $[22,27,28]$ very well. Now we can see that the ground

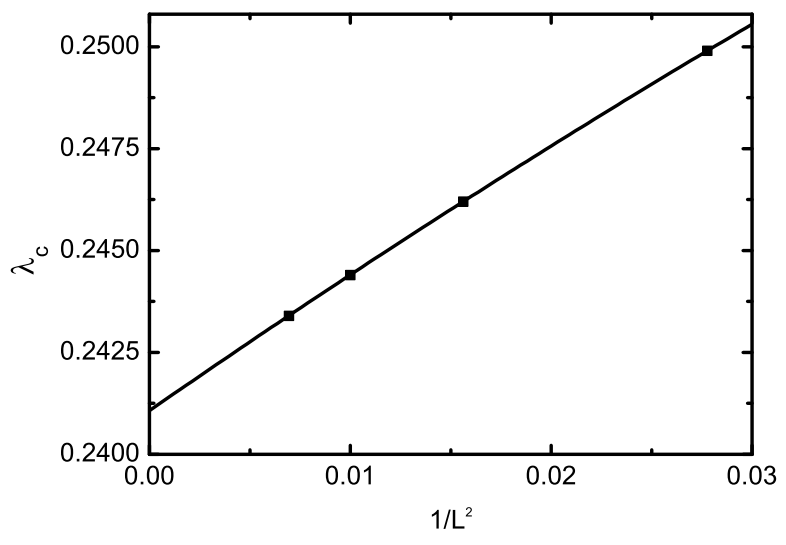

FIG. 4: Finite size scaling of the extrema of first excited state fidelity. A polynomial fit is made. According to this fit, when it comes to the point $L \rightarrow \infty, \lambda_{c}=0.24107$.

state fidelity is not always effective for different models, at least for the $J_{1}-J_{2}$ model. For the model considered in this paper, instead of the ground state fidelity, we need to rely on the first excited state fidelity to characterize the critical points of the quantum phase transition.

To further exemplify why the fidelity of the first excited state instead of the ground state fidelity is able to characterize the QPT for the model considered here, let us consider the lowest energy levels of the model (10) and analyze its implication to the fidelity. In Fig. 15, we plot the energy spectrums of the Hamiltonian with $L=10$ in the regime of $0<\lambda<0.5$. The ground state is a singlet with $S_{\text {total }}^{z}=0$ and is non-degenerate except for the Majumda-Ghosh point with $\lambda=0.5$. The excited states corresponding to the dashed line are three-fold degenerate triplet with $S_{\text {total }}^{z}=0, \pm 1$, whereas the state corresponding to the dotted line is a singlet. It is clear that no level crossing occurs for the ground state energy. In general, the first order quantum phase is characterized by the ground state level crossing which leads to the singularity of ground state fidelity around to the crossing point. Therefore the ground state fidelity is a natural choice for characterizing the first order QPT. When the level crossing of the ground state is absent, the continuous quantum phase transitions are actually caused by a reconstruction (level crossing) of low-excitation spectrum of the system [29]. Therefore for such kind of system, the fidelity of the first-excited state might be a better indicator of QPT. The level crossing of the excited state implies that the corresponding fidelity will suddenly drop to zero in the crossing point. This gives a straightforward explanation for why the fidelity of the excited state is a suitable indicator for the QPT of the $J_{1}-J_{2}$ model.

Though we restrict our attention to the $J_{1}-J_{2}$ model, the similar property can be found in the BKT-like QPTs 


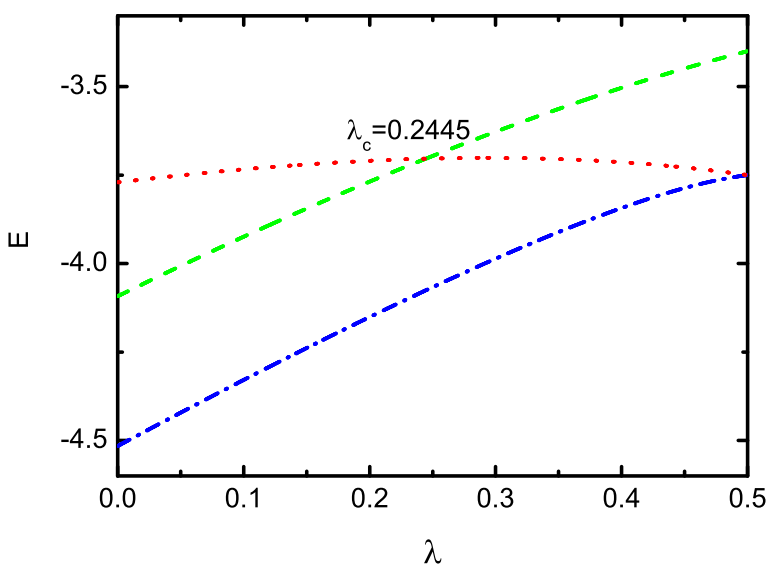

FIG. 5: (Color online). The energy spectrum of a Heisenberg chain with $L=10$. Only the lowest three energy levels are given above. The first and the second excited energy levels cross each other at the point $\lambda_{c}=0.2445$. On the left of $\lambda_{c}$, the first excited energy level is triply degenerate and on the right, it's a singlet.

of other models, such as the one-dimensional anisotropic Heisenberg model whose Hamiltonian reads

$$
H(\Delta)=\sum_{j=1}^{L}\left(\hat{s}_{j}^{x} \hat{s}_{j+1}^{x}+\hat{s}_{j}^{y} \hat{s}_{j+1}^{y}+\Delta \hat{s}_{j}^{z} \hat{s}_{j+1}^{z}\right)
$$

For the anisotropic Heisenberg model, a BKT-like phase transition happens at the point $\Delta=1$, which is described by a divergent correlation length but without true long- range order. However, like the case happened in $J_{1}-J_{2}$ model, the fidelity induced by the anisotropic term does not show the desired singularity at the critical point. This phenomenon is consistent with the fact that the ground state fidelity intrinsically depends on the fluctuation of the driving term [12], and such a fluctuation shows no singularity because of the absence of true long-range order around the critical point. On the other hand, like $J_{1}-J_{2}$ model, the phase transition in the anisotropic Heisenberg model is also induced by the first excited state level-crossing [29]. This fact leads to that the first excited state overlap collapses at the critical point.

In summary, we have calculated the fidelity of the ground state and the first excited state of the spin chain model with the NNN interaction. Our results show that, contrary to the first-order QPT for which the ground state fidelity is a good indicator, the fidelity of the lowlying excited state is an effective tool to quantify the quantum phase transition for the system in which the continuous phase transition is induced by the low-lying excited states. Though we restrict our calculation on the $J_{1}-J_{2}$ model, our observation is general for a class of BKT-like QPTs, which are induced by the first excited state level-crossing, in the other one-dimensional manybody systems, for which the discontinuity of fidelity of the first excited state is intrinsically related to the QPTs.

\section{Acknowledgments}

This work is supported by NSF of China under Grant No. 10574150, programs of Chinese Academy of Sciences, and RGC Grant CUHK 401504. SJ Gu is grateful for the hospitality of Institute of Physics and Theoretical Physics at Chinese Academy of Sciences.
[1] S. Sachdev, Quantum Phase Transitions (Cambridge University Press, Cambridge, England, 1999).

[2] A. Osterloh, Luigi Amico, G. Falci and Rosario Fazio, Nature (London) 416, 608 (2002); T. J. Osborne and M. A. Nielsen, Phys. Rev. A 66, 032110 (2002).

[3] G. Vidal, I. Latorre, E. Rico, and A. Kitaev, Phys. Rev. Lett. 90, 227902 (2003).

[4] S. J. Gu, S. S. Deng, Y. Q. Li and H. Q. Lin, Phys. Rev. Lett. 93, 086402 (2004).

[5] S. J. Gu, H. Li, Y. Q. Li and H. Q. Lin, Phys. Rev. A 70, 052302 (2004).

[6] L. Amico, R. Fazio, A. Osterloh, V. Vedral, arXiv:quant-ph/0703044 [Rev. Mod. Phys. to appear].

[7] R. W. Chhajlany, P. Tomczak, A. Wójcik, and J. Richter, Phys. Rev. A 75, 032340 (2007).

[8] H. T. Quan, Z. Song, X. F. Liu, P. Zanardi, and C. P. Sun, Phys. Rev. Lett. 96, 140604 (2006).

[9] P. Zanardi and N. Paunkovic, Phys. Rev. E 74, 031123 (2006).

[10] P. Buonsante and A. Vezzani, Phys. Rev. Lett. 98,
110601 (2007).

[11] P. Zanardi, M. Cozzini, and P. Giorda, J. Stat. Mech. 2, L02002 (2007); M. Cozzini, P. Giorda, and P. Zanardi, Phys. Rev. B, 75, 014439 (2007); M. Cozzini, R. Ionicioiu, and P. Zanardi, arXiv: cond-mat/0611727.

[12] W. L. You, Y. W. Li, S. J. Gu, Phys. Rev. E 76, 022101 (2007).

[13] H. Q. Zhou, J. P. Barjaktarevic, arXiv: cond-mat/0701608 H. Q. Zhou, J. H. Zhao, B. Li, arXiv:0704.2940 H. Q. Zhou, arXiv:0704.2945.

[14] H. A. Bethe, Z. Physik 71, 205 (1931).

[15] M. Takahashi, Thermodynamics of one-dimensional Solvable Models (Cambridge University Press, Cambridge, 1999).

[16] C. K. Majumdar and D. K. Ghosh, J. Math. Phys. 10, 1388 (1969); C. K. Majumdar and D. K. Ghosh, ibid. 10, 1399 (1969).

[17] F. D. M. Haldane, Phys. Rev. B 25, 4925 (1982).

[18] T. Giamarchi, Quantum Physics in One Dimension (Oxford University Press, Oxford, England, 2004). 
[19] T. Tonegawa, and I. Harada, J. Phys. Soc. Jpn. 56, 2153(1987).

[20] S. Eggert, Phys. Rev. B 54, R9612 (1996).

[21] R. J. Bursill, et al, J. Phys. C 7, 8605 (1995).

[22] R. Chitra, S. Pati, H. R. Krishnamurthy, D. Sen, and S. Ramasesha, Phys. Rev. B 52, 6581 (1995).

[23] S. R. White, and I. Affleck, Phys. Rev. B 54, 9862 (1996).

[24] V. L. Beresinskii, Sov. Phys. JETP 32, 493 (1971).

[25] J. M. Kosterlitz and D. J. Thouless, J. Phys. C 6, 1181 (1973); J. M. Kosterlitz, J. Phys. C 7, 1046 (1974).
[26] I. Affleck, D. Gepner, H. J. Schulz, and T. Ziman, J. Phys. A 22, 511 (1989).

[27] K. Okamto and K. Nomura, Phys. Lett. A 169, 433 (1992).

[28] G. Castilla, S. Chakravarty, and V.J. Emery, Phys. Rev. Lett. 75, 1823 (1995).

[29] G. S. Tian and H. Q. Lin, Phys. Rev. B 67, 245105 (2004). 